\title{
Solar-type cycles of the secondary stars in cataclysmic variables
}

\author{
T. Ak ${ }^{1}$, M. T. Ozkan ${ }^{2}$, and J. A. Mattei ${ }^{3}$ \\ 1 University of Istanbul, Faculty of Science, Department of Astronomy and Space Sciences, \\ 34452, University, Istanbul, Turkey \\ 2 Istanbul University Observatory Research and Application Center, 34452, University, Istanbul, Turkey \\ 3 American Association of Variable Star Observers, 25 Birch Street, Cambridge, MA 02138, USA \\ e-mail: jmattei@aavso.org
}

Received 16 November 1999 / Accepted 19 December 2000

\begin{abstract}
We found cyclical variations in the quiescent magnitudes and outburst intervals of 21 dwarf novae, 1 nova and 1 nova-like star produced by the solar-type cycles of the late-type secondary components. More than $70 \%$ of individual systems in this work are first studied by Fourier periodogram analysis. It seems that the mean cycle amplitudes of the quiescent magnitudes are considerably larger for SU UMa stars. Cyclical modulations of quiescent magnitudes found for the stars, which have orbital periods below the period gap, support the hypothesis that even almost fully convective secondaries may possess activity cycles. The estimated probability density functions show peaks at 9.7, 7.9 and 8.6 yrs for cataclysmic variables (CVs), single main-sequence stars (MS) and all stars (CVs+MS), respectively. Using a larger set of data, we confirm that there is no correlation of the cycle period with the rotational regime of the secondary star. It is also confirmed that cycle periods of CVs are in the range of those of single main-sequence stars. We note that the observational cycle parameters (i.e. cycle period, $P_{\text {cyc }}$, and cycle amplitude of quiescent magnitudes, $\Delta m$ ) do not seem in relation with any other parameter such as masses of primary and secondary stars, mean outburst interval, mean outburst duration, mean decline and rise rate of outbursts, absolute magnitudes in quiescent and outburst states etc.
\end{abstract}

Key words. stars: cataclysmic variables - stars: activity

\section{Introduction}

Cataclysmic variables (hereafter referred to as CVs) are short period binary stars in which a Roche lobe-filling, lower main-sequence star, the secondary star, transfers matter onto a white dwarf through an accretion disk. A hot spot is created where the mass flow strikes the accretion disk.

Classification of CVs is based on the characteristics of the long-term visual light curves. These stars are classified into four types: novae, dwarf novae, nova-like stars and recurrent novae. Novae have had outburst only once within our recorded history, in which amplitudes ranged from 8 to 18 magnitudes. They are divided into the categories fast, moderate and slow, depending upon the rate of decline of 2 magnitudes from maximum. Dwarf novae are characterized by quasi-periodic outbursts occuring every 10 to 500 or more days, with amplitudes of 2-6 magnitudes. Dwarf novae are classified into three sub-types: U Geminorum (U Gem) stars have only normal outbursts, being brightenings of 2-6 magnitudes from the quiescent state. In ad-

Send offprint requests to: T. Ak, e-mail: tanselak@istanbul.edu.tr dition to normal outbursts, Z Camelopardalis (Z Cam) stars have standstills lasting from a few weeks to years, during which outburst behaviour disappeares and the system is observed in a high state which is brighter than the quiescent phase. SU Ursae Majoris (SU UMa) stars show high amplitude, wide outbursts (superoutbursts), in addition to normal outbursts. Nova-like stars do not show eruptive activity, and in fact some even have antieruptions during which the star fades in brightness by 2-5 magnitudes. Recurrent novae have outbursts more than once. Amplitude of the outbursts of recurrent novae ranges from 7 to 9 magnitudes (Mattei 1990).

Quasi-periodic outburst behaviour of dwarf novae is more easily explained by viscous processes acting in the accretion disk, instead of mass transfer variations originating from evolutional state or dynamical instabilities of the secondary star (Cannizzo et al. 1988; Cannizzo 1993; Hameury et al. 1998).

Roche-lobe filling secondary stars of CVs are nearly identical to single lower main-sequence stars (Warner 1995; Beuermann 1998). With their deep convective zones and high rotation rate synchronized with orbital period, secondaries of CVs suggest the existence of solar-type 
cyclical magnetic activity. Warner (1988) has demonstrated that the variation of the quadrupole moment caused by a solar-type magnetic cycle produces the cyclical variations of some observable parameters of CVs. Increasing the number of magnetic flux tubes causes an increase in radius of the star (or a decrease in Roche lobe radius, Richmann 1994), resulting in an enhancement of mass transfer. Enhanced mass transfer gives rise to an increase in hot spot luminosity and mass flow rate through the accretion disk. Thus, the total luminosity of the system is increased in the quiescent phase, because the dominant sources of the quiescent light are the secondary star, the hot spot and the accretion disk. Since the outburst interval (time interval between consecutive outbursts) of dwarf novae is inversely correlated with the mass transfer rate (Ichikawa \& Osaki 1994), enhanced mass transfer rates make the outburst intervals shorter. The magnetic field of the secondary would reach over the accretion disk. Magnetic field lines penetrate the disk and remove angular momentum, and the region where mass accumulates and outbursts are triggered shifts towards inner parts of the disk. Then, outbursts are expected to be narrower and more frequent (Vogt et al. 1995; Smak 1984). In short, cyclical magnetic activity of the secondary star in a cataclysmic variable can be observed as cyclical variations in brightness of the system (for all types of $\mathrm{CVs}$ ), in the outburst intervals and the outburst durations of dwarf novae.

Since the distribution of angular momentum in the secondary star changes as the star goes through its activity cycle, variations in the distribution of angular momentum produce variations in the oblateness of the star. These changes are communicated to the orbit by gravity, changing the orbital period (Applegate 1992; Warner 1988).

Observational tests of magnetic activity cycles in the secondary stars of CVs can be performed by analysing long-term observations of the systems. Indeed, such cycles were found by Bianchini $(1988,1990)$ in long-term visual magnitudes of some novae and nova like-stars, and in outburst intervals of some dwarf novae. Although their sample is limited to a small number of CVs, Bianchini (1990) and Maceroni et al. (1990, and references therein) found that cycle periods of CVs, RS CVn and W UMa stars are in the range of those of single main-sequence stars, and that there is no correlation of the cycle period with the rotational regime of the secondary star.

Although magnetic cycles of the single stars have been detected (Baliunas et al. 1995; Wilson 1978), cataclysmic variables have some advantages when working on magnetic activity cycles. First, long-term visual observations made by amateur astronomers have been compiled and collected by some associations for decades, for example AAVSO (American Association of Variable Star Observers) and RASNZ (Royal Astronomical Society of New Zealand). Second, since the orbital period is directly correlated to basic stellar parameters such as stellar rotation, mass, radius etc., and orbital period is the best determined parameter of CVs, stellar parameters are determined to an acceptable accuracy for some of them.
Table 1. Names, sub-types, observation intervals, number of quiescent observations $\left(N_{\mathrm{q}}\right)$, cycle periods $\left(P_{\text {cyc }}\right)$, and cycle amplitudes of quiescent magnitudes ( $\Delta m$, peak to peak range) for stars studied here. Observations of SS Cyg, SS Aur and AH Her consist of daily means. Dwarf novae are listed according to the sub-type. $U G$ denotes $U$ Gem type dwarf novae while $Z C$ Z Cam type, and $S U$ SU UMa type dwarf novae. $N$ and $N L$ denote members of novae and nova-like stars in our sample, respectively

\begin{tabular}{lccccc}
\hline System & Sub-type & $\begin{array}{c}\text { Interval } \\
\text { (years) }\end{array}$ & $N_{\mathrm{q}}$ & $\begin{array}{c}P_{\text {cyc }} \\
\text { (years) }\end{array}$ & $\begin{array}{c}\Delta m \\
(\mathrm{mag})\end{array}$ \\
\hline BV Pup & UG & $1978-1997$ & 1758 & $14.2 \pm 0.6$ & 0.21 \\
CZ Ori & UG & $1978-1997$ & 1006 & $17.1 \pm 0.9$ & 0.30 \\
FQ Sco & UG & $1972-1997$ & 4632 & $8.6 \pm 0.7$ & 0.12 \\
SS Aur & UG & $1961-1997$ & 5194 & $10.6 \pm 0.6$ & 0.17 \\
SS Cyg & UG & $1896-1992$ & 20064 & $7.2 \pm 0.4$ & 0.11 \\
UZ Ser & UG & $1976-1997$ & 3380 & $6.7 \pm 0.6$ & 0.15 \\
V442 Cen & UG & $1973-1997$ & 9296 & $8.0 \pm 0.5$ & 0.11 \\
U Gem & UG & $1979-1995$ & 10512 & $5.6 \pm 0.7$ & 0.13 \\
TT Crt & UG & $1989-1997$ & 426 & $4.3 \pm 0.9$ & 0.23 \\
AH Her & ZC & $1969-1995$ & 2343 & $16.0 \pm 0.8$ & 0.15 \\
HL CMa & ZC & $1981-1997$ & 542 & $2.8 \pm 0.1$ & 0.22 \\
UY Pup & ZC & $1984-1997$ & 1258 & $5.7 \pm 0.3$ & 0.13 \\
WW Cet & ZC & $1979-1997$ & 2013 & $5.6 \pm 0.3$ & 0.14 \\
Z Cam & ZC & $1963-1995$ & 10340 & $19.9 \pm 0.7$ & 0.20 \\
EK TrA & SU & $1973-1997$ & 3829 & $8.9 \pm 0.6$ & 0.20 \\
SW UMa & SU & $1962-1997$ & 3172 & $13.6 \pm 1.3$ & 0.32 \\
TU Men & SU & $1979-1997$ & 1678 & $8.2 \pm 0.8$ & 0.32 \\
WZ Sge & SU & $1979-1997$ & 3431 & $10.6 \pm 0.5$ & 0.31 \\
OY Car & SU & $1979-1997$ & 2972 & $6.3 \pm 0.6$ & 0.26 \\
Z Cha & SU & $1979-1997$ & 4195 & $14.6 \pm 0.4$ & 0.16 \\
V436 Cen & SU & $1972-1997$ & 1686 & $26.3 \pm 0.8$ & 0.57 \\
GK Per & N & $1961-1997$ & 14092 & $27.1 \pm 0.8$ & 0.15 \\
EX Hya & NL & $1954-1997$ & 14712 & $28.7 \pm 0.9$ & 0.16 \\
\hline
\end{tabular}

Here, we concentrate on the long-term visual light curves of some dwarf novae, one nova and one nova-like star in order to detect the long-term variabilities which can be considered as diagnostics of the presence of solar-type magnetic activity cycles in their secondary stars. Since the number of known CVs showing magnetic cycles is small for a study of relations between observable cycle parameters and other system parameters, such as orbital period, outburst duration, outburst intervals etc., this work also increases the number of such systems.

\section{Data and analyses}

The data sample consists of long-term visual observations of 21 dwarf novae, 1 nova (GK Per) and 1 nova-like star (EX Hya) taken from the AAVSO and RASNZ. GK Per shows dwarf nova-type outbursts. Durations of the light curves are between 8 (TT Crt) and 96 (SS Cyg) years.

Quiescent magnitudes, which are given in unequally spaced time intervals, are selected from the long-term visual light curves and analysed by Fourier transform. While working on such data files, irregular and regular spacings, which originate from nature of the 

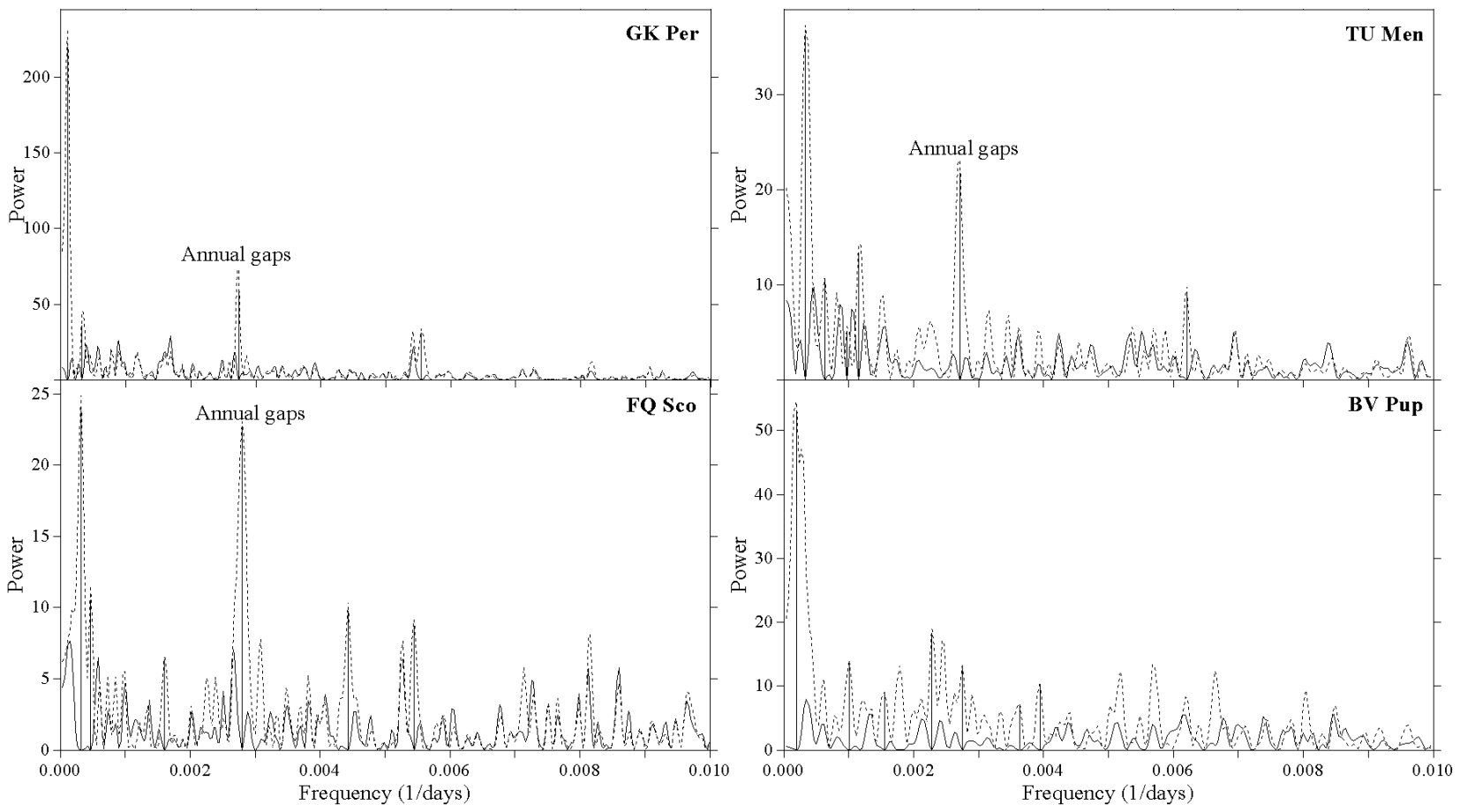

Fig. 1. Examples from power spectra (dashed line) and cleanest spectra (solid line) of stars studied in this work. Main signals at $0.000101,0.000335,0.000321$ and 0.000193 days $^{-1}$ are clearly visible for GK Per, TU Men, FQ Sco and BV Pup, respectively. For the other stars, power spectra are not much different to those shown here, except in the location of main signals. Signals originating from annual gaps in observations are also shown on the power spectra

observations, removing of outburst data and obscuration of the stars by the sun, complicates the Fourier transform. To deal with this problem, astronomers use a modified periodogram (Scargle 1982), which is based on a leastsquares regression onto two trial functions, $\sin (\omega t)$ and $\cos (\omega t)$. Here, we used a lesser-known but superior technique developed (Foster 1995) specifically to apply Fourier analysis to long-term visual data of variable stars from International Databases such as AAVSO and RASNZ, in order to remove the false peaks. This newer technique is based on Data Compensated Discrete Fourier Transform (DCDFT, Ferraz-Mello 1981), a least-square regression on $\sin (\omega \mathrm{t})$ and $\cos (\omega \mathrm{t})$, and a constant. This technique also includes the CLEAN algorithm (Roberts et al. 1987).

By the beginning of the work, in fact, we had longterm visual observations of 40 systems. Earlier observations were removed from data sets for some systems, since we encountered very wide gaps in data files, especially before JD 2440000. As a first step, only those peaks of the power spectrum whose height was above the noise level at a $>95 \%$ confidence level were considered. Therefore, we quantitatively estimated the statististical significances of peaks in the periodograms (see, Scargle 1982; Horne \& Baliunas 1986) and found that in almost all cases statistical confidences of peaks, which are selected as indicators of real signals, are near $99 \%$.

We then applied the CLEAN algorithm to remove the false peaks until the strongest residual peak is below a given cutoff level. Some examples of the power spectra of

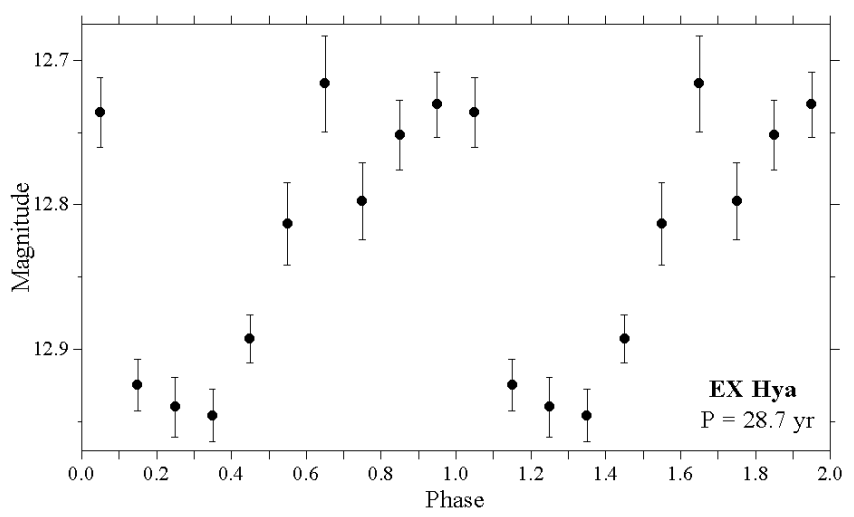

Fig. 2. Folded light curve of the nova-like star EX Hya with the period found by Fourier analysis of its long-term visual observations. The folded data have been averaged over bins of $0.1 P_{\text {cyc }}$. Each symbol represents 158 averaged observations over 5 days. Standard deviations of mean values are shown by bars

the stars studied in this work are shown in Fig. 1. The second step was to remove the systems having less than one complete cycle and/or having amplitudes smaller than 0.1 magnitude for the sake of confidence in the analysis and the observations. At the third step, all data points were averaged over different time intervals for each system, such as 10 days, 100 days, 500 days. Then the averaged data were Fourier analysed by means of the same technique mentioned above. 


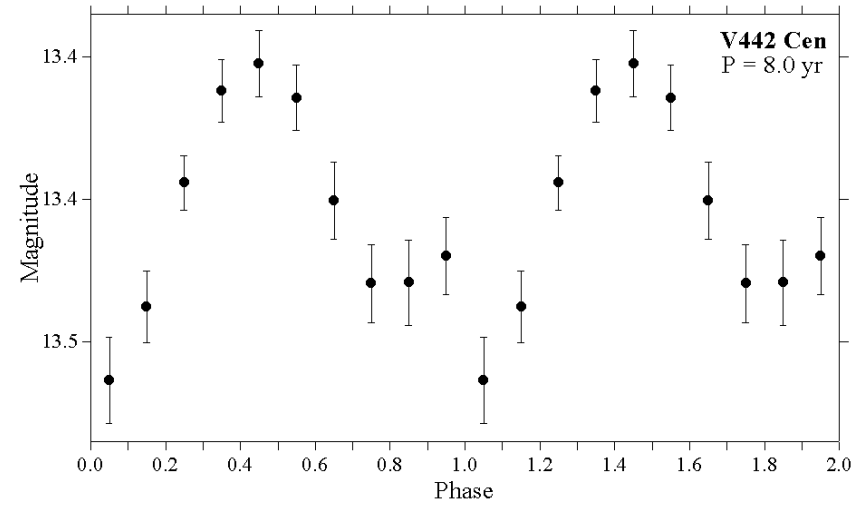

Fig. 3. Folded light curve of the U Gem-type dwarf nova, V442 Cen, with the period found by Fourier analysis. Mean values and error bars are as in Fig. 2. Each symbol represents 270 observations

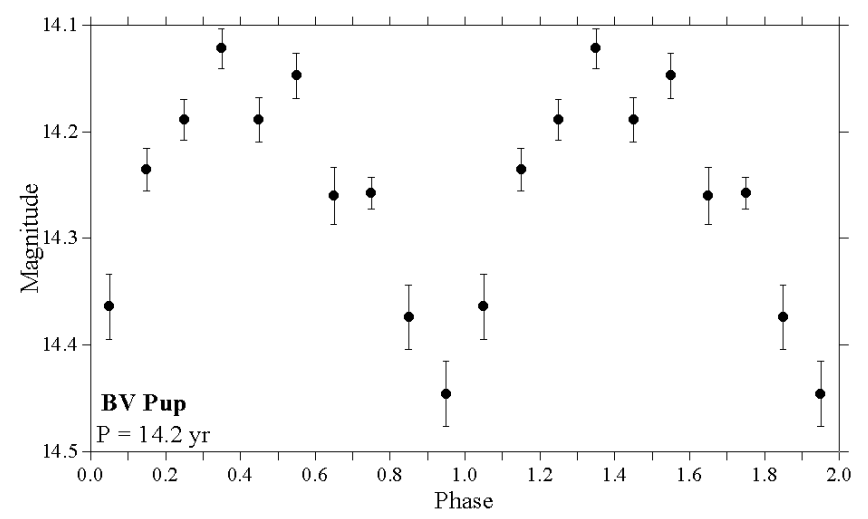

Fig. 4. Folded light curve of the U Gem-type dwarf nova, BV Pup, with the period found by Fourier analysis. Mean values and error bars are as in Fig. 2. Each symbol represents 126 observations

Sometimes, relatively short time spans of the quiescent magnitudes implies that only very few cycles are available. However, since the variability that we observe must be connected with the long-term variability of the mass transfer rate from the secondary star, we can simply assume that this mechanism always has a cyclical nature. According to the evidence that we have found, the validity of this assumption is supported (see also Bianchini 1990).

Long-term trends in the data can also be found by moving averages. Thus, in the fourth step, we calculated moving averages of each system over different time intervals. Then we applied polynomial fits to the moving averages and compared them to the sinusoids found from Fourier analysis. Finally, the number of systems for which the cycle periods are indicated by all steps of the analysis was reduced to 23 . The systems, sub-types and observation intervals studied in this work, number of quiescent magnitudes $\left(N_{\mathrm{q}}\right)$, cycle periods $\left(P_{\text {cyc }}\right)$ and cycle amplitudes of quiescent magnitudes $(\Delta m$, peak to peak range) are listed in Table 1 for these 23 system.

We also analysed outburst intervals $(\Delta t)$ of dwarf novae by using the method mentioned above. Although the

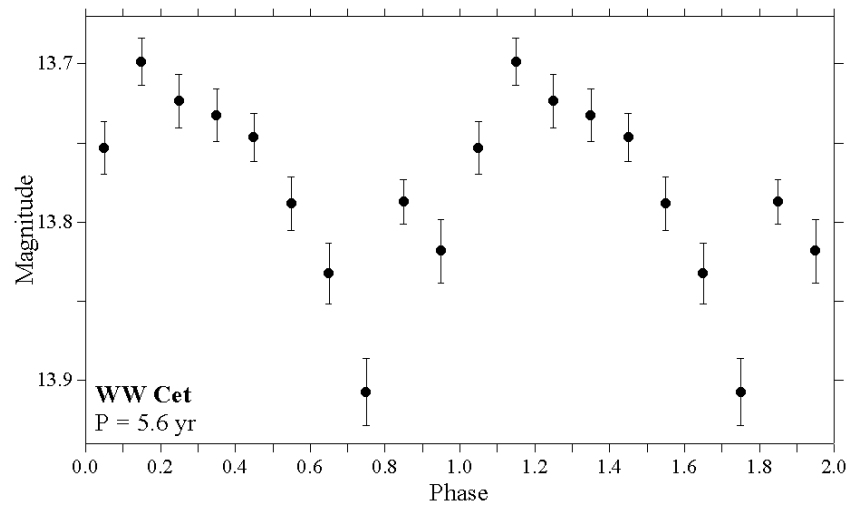

Fig. 5. Folded light curve of the Z Cam-type dwarf nova, WW Cet, with the period found by Fourier analysis. Mean values and error bars are as in Fig. 2. Each symbol represents 195 observations

time span of observations is quite wide in most cases, the number of $\Delta t^{\prime} \mathrm{s}$, which are measured by us for wellobserved outbursts, is too small for most of the systems. This situation reduces the statistical confidence of the results found from analysis. For stars for which such analysis could be done, results obtained from outburst intervals and outburst durations support the previous periodicities we found from the quiescent magnitudes.

In order to represent the mean behavior of quiescent magnitudes, we folded the data with the cycle period found from analysis, and averaged them over bins corresponding to one tenth of the period (Figs. 2-6). Thus, we tentatively derived standard deviations of the means, assuming a random distribution of the fluctuations about the mean values.

In correlation analysis, we follow the approximation made by Cannizzo \& Mattei (1992) based on the complementary error function given by Press et al. (1986, see p. 485) in order to assign a level of significance to the correlation coefficient. So, for $N=20$, values of $|r| \geq 0.22$ are probably strongly indicative of a correlation, since $N^{-1 / 2} \cong 0.22$. By considering that in some cases $N$ drops below 20 , for the sake of confidence, we accept this value as 0.25 and multiply it by 2 . Thus, we find that $|r|$ must be $\geq 0.50$ to conclude the presence of a correlation. We emphasize that the number of systems showing solar-type cycles must be increased to achieve a more reliable correlation analysis.

\section{Remarks and comparisons for some individual stars}

Some stars in this work have been studied previously by other researchers. In this section we give a comparison with previous studies. Those stars studied for the first time are indicated.

GK Per: Although Bianchini (1990) finds a $7.24 \mathrm{yr}$ modulation of its magnitude between 1920 and 1986, this period is only weakly detected by DCDFT in the light curve lasting from 1961 to 1997 studied here. However, 


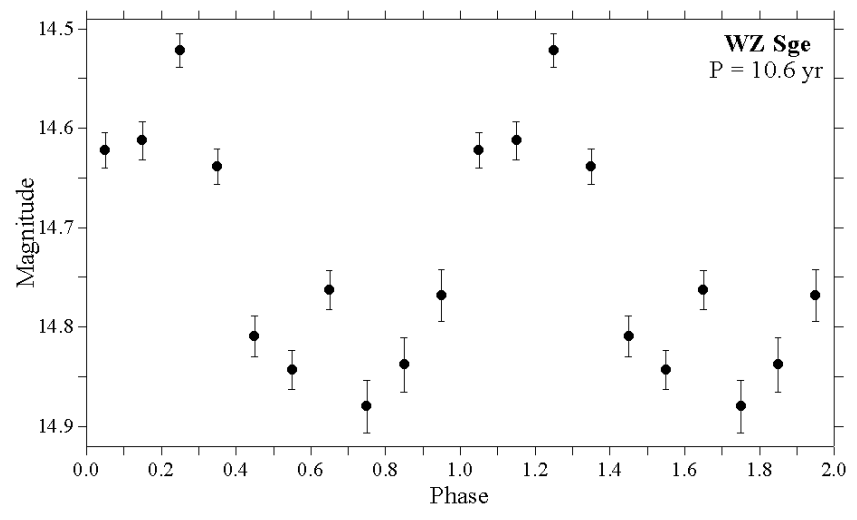

Fig. 6. Folded light curve of the SU UMa-type dwarf nova, WZ Sge, with the period found by Fourier analysis. Mean values and error bars are as in Fig. 2. Each symbol represents 216 observations

DCDFT detected a very clear 27.1 yr modulation in the light curve.

EX Hya: We found a variation with $P=28.7 \mathrm{yr}$ in the long-term visual light curve of this member of novalike stars covering a time interval of $43 \mathrm{yr}$. Warner (1988) suggests a cycle of about $19 \mathrm{yr}$ for EX Hya. His conclusion is based on the presence of an $\sim 19$ yr cyclical variation in orbital period of the system (Bond \& Freeth 1988).

SS Aur: We analysed the long-term visual light curve of SS Aur covering a $36 \mathrm{yr}$ time interval. The periodicities of 2.32 and $1.2 \mathrm{yr}$ given by Bianchini (1990) could not be confirmed in our analysis, even when we used outburst intervals. Instead, we found a $10.6 \mathrm{yr}$ cyclical modulation of the quiescent magnitudes which is clearly seen in the power spectrum. DCDFT do not show clear evidence for any periodicity for outburst intervals.

SS Cyg: This U Gem-type dwarf nova is one of the most studied stars. We analysed the quiescent magnitudes and outburst intervals of SS Cyg selected and measured, respectively, from the long-term visual light curve covering $96 \mathrm{yrs}$. DCDFT gives a $7.2 \mathrm{yr}$ modulation of the quiescent magnitude. Bianchini (1990) found variations in the outburst intervals with $P=7.3 \mathrm{yr}$. It is significant that both Kiplinger et al. (1988) and we find about the same period from the quiescent magnitudes. However, there is also a variation in the quiescent magnitudes with $P=46.7 \mathrm{yr}$. The $7.2 \mathrm{yr}$ modulation is superposed on this long-term variation and the total amplitude is $0.23 \mathrm{mag}$. It is interesting to note that we obtained about the same periods from the outburst intervals $(P=7.2 \mathrm{yr})$ and the outburst durations $(P=7.0 \mathrm{yr})$ of SS Cyg. This result is a clear confirmation of the discussion given in Sect. 1.

$U$ Gem: Although DCDFT detected a strong periodicity of annual gaps $(P=1.00 \mathrm{yr})$, a periodicity of $P=5.6$ yr is clearly present in the quiescent magnitudes. Bianchini (1990) found a variation in the outburst intervals with a similar period of $P=6.9 \mathrm{yr}$.

$R X$ And and RUPeg: While DCDFT finds periodicities in both quiescent magnitudes and outburst intervals of some stars, we could not detect any cyclic variability for these parameters of RX And and RU Peg. However, Bianchini (1990) found a variation with $P=0.88 \mathrm{yr}$ for outburst intervals of RX And, and with $P \approx 6$ and $P \approx 12$ yr for outburst intervals of RU Peg.

$Z$ Cam: We analysed a $32 \mathrm{yr}$ interval of the long-term visual light curve of $\mathrm{Z}$ Cam. The cycle period with $P=$ 19.9 yr given by DCDFT differs from the period $(P=$ $8.84 \mathrm{yr}$ ) found by Bianchini (1990) who studied a light curve covering only $11.4 \mathrm{yr}$.

AH Her: The quiescent magnitudes of this $\mathrm{Z}$ Cam type star shows a cyclical variation with $P=16.0 \mathrm{yr}$. Outburst intervals and outburst durations follow this variation: fainter quiescent states of the system correspond to longer outburst intervals and longer outburst durations.

UZ Ser: DCDFT gives a general increase in the quiescent magnitudes for UZ Ser, while it also gives a cyclical variation with $P=6.7$ yr. Annual gaps are apparent in the power spectrum.

V442 Cen: DCDFT gives a general increase in the quiescent magnitudes also for this star, in addition to the cyclical variability of $P=8.0 \mathrm{yr}$. Behavior of outburst intervals and outburst durations resemble those we obtained for AH Her.

TT Crt: Although the number of quiescent observations is relatively small, an apparent cyclical modulation of the quiescent magnitudes with $P=4.3 \mathrm{yr}$ is detected by DCDFT.

SU UMa stars: According to Warner (1988) and Bianchini (1990), cycles of the very short orbital period $\left(P_{\text {orb }}=1.64 \mathrm{hr}\right.$, i.e., below the period gap $)$ nova-like star, EX Hya, would demonstrate that even almost fully convective secondaries may possess cycles of activity. Since SU UMa stars are below the period gap, cycles found in the long-term visual light curves of these stars support this hypothesis. In this study, we found cyclical variations in the quiescent magnitudes of $7 \mathrm{SU}$ UMa stars.

\section{Results and discussion}

Although, at first, we had data for 40 systems, we have detected long-term cycles in the long-term visual quiescent magnitudes of 21 dwarf novae, 1 nova and 1 nova-like star among them. The reasons for this likely are: (i) low amplitude of the variations; (ii) the presence of irregular fluctuations of the mean activity level (this occured to the Sun in the Maunder minimum of the late 17th century during which cyclic activity of the Sun varied in strenght and shape, and even disappeared for several cycles); (iii) the short time base of the data. The signal to noise ratio, which can be seen also by an inspection of Figs. 2-6, is high enough to state the presence of cycles of CVs. Even in the case in which only one cycle was analysed, the cyclical nature of the visual magnitude appears rather certain. The presence of long-term periodicities in 4 more nova-like stars and 6 more novae is shown in the literature (see Bianchini 1990; Maceroni et al. 1990, and references therein). 


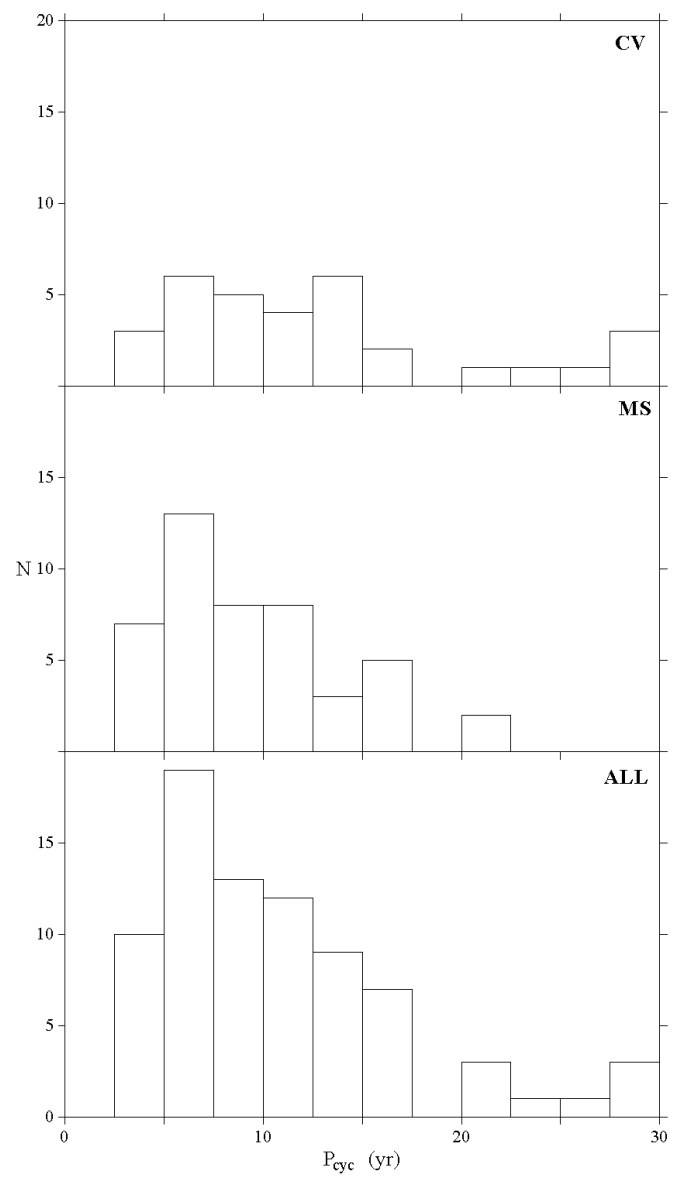

Fig. 7. Period histograms for cataclysmic variables (CV) and single main-sequence stars (MS). The estimated probability density functions show peaks at 9.7, 7.9 and 8.6 yrs for cataclysmic variables, single main-sequence stars and all stars, respectively

Thus, in this study, we show that cycle periods of dwarf novae can be detected from quiescent magnitudes. However, it is possible to confirm these periods with those found from outburst intervals and from outburts durations (e.g. SS Cyg, AH Her, V442 Cen) This result is a clear observational confirmation of the discussion given in Sect. 1 .

We only confirm the cycle periods of SS Cyg and U Gem obtained by Bianchini (1990) from outburst intervals of these systems. Although we worked both on outburst intervals and quiescent magnitudes of the dwarf novae which are also studied by Bianchini, we do not confirm previous cycle periods given for 4 dwarf novae, 1 nova and 1 nova-like star. The reason for the difference of results between previous and this work may be due to: (i) the small amplitude of the cycle in the quiescent magnitudes, (ii) the short time base of the data used in previous work, resulting in a small number of outburst intervals, (iii) inhomogenity of outburst interval data sets which are used in Bianchini's work, since they are measured by various researchers. This last item is crucial when we study the long-term light curve parameters of dwarf novae.

More than $70 \%$ of individual systems listed in Table 1 are first studied by Fourier periodogram analysis via
DCDFT. About half of the systems studied in this work have cycle amplitudes $\geq 0.20 \mathrm{mag}$ (BV Pup, CZ Ori, TT Crt, HL CMa, Z Cam, EK TrA, SW UMa, TU Men, WZ Sge, OY Car, V436 Cen). These systems may have special interest also in their orbital period modulations, since variations in the oblateness of the secondary star due to changes in the distribution of angular momentum can produce orbital period modulations (Applegate 1992).

Mean cycle amplitudes of quiescent magnitudes are 0.17, 0.17 and $0.31 \mathrm{mag}$ for U Gem, Z Cam and SU UMa type dwarf novae, respectively. Since a higher amplitude of the cycle means a larger difference in mass transfer rates between minimum and maximum phases of the cycle, it is possible to say that mass transfer changes of $\mathrm{SU}$ UMa stars, which are caused by solar-type magnetic cycles, are stronger than those of other types of dwarf novae. In this study, we found cyclical modulations in the quiescent magnitudes of 7 SU UMa stars and of EX Hya which have orbital periods below the period gap. These results support the hypothesis given by Bianchini (1990) and Warner (1988) so that even almost fully convective secondaries may possess activity cycles.

We can use the data provided by Baliunas et al. (1995) on the long-term variations of the CaII H and K chromospheric emissions from main-sequence stars, in order to compare the cycle periods found in CVs with those observed in single main-sequence stars. Observations of almost all main-sequence stars cover about a 24 yr interval. Cycle periods of single stars have been choosen according to their primary classification in Baliunas et al. (1995), including all excellent, good, fair and poor FAP Grades. Distributions of the cycle periods of CVs, main-sequence stars and all stars $(\mathrm{CVs}+\mathrm{MS})$ are given in Fig. 7. The estimated probability density functions show peaks at 9.7, 7.9 and 8.6 yrs for cataclysmic variables, single main-sequence stars and all stars, respectively.

Finally, we note that the linear correlation analysis does not show a clear correlation between cycle parameters (i.e. cycle period, $P_{\text {cyc }}$, and cycle amplitude of quiescent magnitudes, $\Delta m$ ), and other system parameters, such as orbital period $\left(P_{\text {orb }}\right)$, masses of primary $\left(M_{1}\right)$ and secondary $\left(M_{2}\right)$ stars. System parameters are taken from Ritter \& Kolb (1995). Thus, we confirm that there is no correlation of the cycle period with the rotational regime of the secondary star, since rotation of the secondary star is syncronized with the orbital period (Patterson 1984), as Maceroni et al. (1990) previously noted. We also find that cycle parameters are not clearly correlated with other observational parameters, such as outburst interval $\left(t_{c}\right)$, outburst duration $\left(t_{\mathrm{b}}\right)$, absolute magnitudes in quiescent and outburst states etc. taken from Warner (1987), Szkody \& Mattei (1984), and Bruch \& Engel (1994).

Acknowledgements. We acknowledge the observers of the AAVSO and RASNZ who made the thousands of observations that comprise the data set used in this study. Thanks are due to Dr. F. M. Bateson for his help in obtaining the data. We also thank to Dr. E. Budding. We wish to thank the anonymous 
referee for his/her comments and suggestions on an earlier draft of this manuscript. Part of this work was supported by the Research Fund of the University of Istanbul, Project Number $\mathrm{T}-141 / 241095$.

\section{References}

Applegate, J. H. 1992, ApJ, 385, 621

Baliunas, S. L., Donahue, R. A., Soon, W. H., et al. 1995, ApJ, 438,269

Beuermann, K., Baraffe, I., Kolb, U., \& Weichhold, M. 1998, A\&A, 339, 518

Bianchini, A. 1988, IBVS, No. 3136

Bianchini, A. 1990, AJ, 99, 1941

Bond, I. A., \& Freeth, R. V. 1988, MNRAS, 232, 753

Bruch, A., \& Engel, A. 1994, A\&AS, 104, 79

Cannizzo, J. K., Shafter, A. W., \& Wheeler, J. C. 1988, ApJ, 333,227

Cannizzo, J. K., \& Mattei, J. A. 1992, ApJ, 401, 642

Cannizzo, J. K. 1993, ApJ, 419, 318

Ferraz-Mello, S. 1981, AJ, 86, 619

Foster, G. 1995, AJ, 109, 1889

Hameury, J. M., Menou, K., Dubus, G., Lasota, J. P., \& Hure, J. M. 1998, MNRAS, 298, 1048

Horne, J. H., \& Baliunas, S. L. 1986, ApJ, 302, 757

Ichikawa, S., \& Osaki, Y. 1994, in Theory of Accretion Disks2, ed. W. J. Duschl, J. Frank, F. Meyer, E. MeyerHofmeister, \& W. M. Tscharnuter (Kluwer Academic Publishers, Dordrecht), 169
Kiplinger, A. L., Mattei, J. A., Danskin, K. H., \& Morgan, J. E. 1988, J. Am. Assoc. Variable Star Obs., 17, 34

Maceroni, C., Bianchini, A., Rodono, M., Van't Veer, F., \& Vio, R. 1990, A\&A, 237, 395

Mattei, J. A. 1990, Optical Properties of Cataclysmic Variable Stars, in ed. C. Ibanoglu, Proc. of the NATO ASI on Active Close Binaries, Active Close Binaries (Kluwer, Dordrecht), 611

Patterson, J. 1984, ApJS, 54, 443

Press, W. H., Flannery, B. P., Teukolsky, S. A., \& Vetterling, W. T. 1986, Numerical Recipes (Cambidge Univ. Press, New York)

Richmann, H. R., Applegate, J. H., \& Patterson, J. 1994, PASP, 106, 1075

Ritter, H., \& Kolb, U. 1995, in X-ray Binaries, ed. W. H. G. Lewin, J. van Paradijs, \& E. P. J. van den Heuvel (Cambridge Univ. Press, Cambridge), 578

Roberts, D. H., Lehar, J., \& Dreher, J. W. 1987, AJ, 93, 968

Scargle, J. 1982, ApJ, 263, 835

Smak, J. 1984, Acta Astron., 34, 161

Szkody, P., \& Mattei, J. A. 1984, PASP, 96, 988

Vogt, N., Meyer-Hofmeister, E., \& Meyer, F. 1995, in Proc. of IAU Coll. No. 151, Flares and Flashes, ed. J. Greiner, H. W. Duerbeck, \& R. E. Gershberg (Springer-Verlag, Berlin), 285 Warner, B. 1987, MNRAS, 227, 23

Warner, B. 1988, Nature, 336, 129

Warner, B. 1995, Ap\&SS, 232, 89

Wilson, O. C. 1978, ApJ, 226, 379 